\title{
Ray-based description of normal mode amplitudes in a range-dependent waveguide
}

\author{
A.L. Virovlyansky, A.Yu. Kazarova, L.Ya. Lyubavin \\ Institute of Applied Physics, 46 Ulyanova St., Nizhny Novgorod, 603950 Russia
}

\begin{abstract}
An analogue of the geometrical optics for description of the modal structure of a wave field in a range-dependent waveguide is considered. In the scope of this approach the mode amplitude is expressed through solutions of the ray equations. This analytical description accounts for mode coupling and remains valid in a nonadiabatic environment. It has been used to investigate the applicability condition of the adiabatic approximation. An applicability criterion is formulated as a restriction on variations of the action variable of the ray.

Key words: waveguide, ray, mode, coupling, adiabatic approximation, action-angle variables
\end{abstract}

\section{INTRODUCTION}

We consider a method for analysis of the modal structure of a scalar wave field in a two dimensional rangedependent waveguide. This method derived in Refs. [13] (see also [4]) is based on projecting the ray representation of the wave field onto normal modes and evaluating the corresponding integrals using the stationary phase technique. As a result the mode amplitude is expressed through parameters of ray trajectories. This approach establishes a relationship between the ray and mode representations of the wave field in a range-dependent waveguide and provides an approximate solution to the mode coupling equation.

In describing ray trajectories we apply the Hamiltonian formalism taken in terms of the action-angle variables $[5,6]$. It is shown that each mode is formed by contributions from rays whose action variables up to a multiplicative constant are equal to the mode number. In the nonadiabatic environment the action varies along the ray path. Our objective in this work is to find out how the nonadiabaticity of rays manifests itself in variations of mode amplitudes. In the nonadiabatic waveguide an initially excited single mode breaks up into a group of modes. We argue that the width of the group is proportional to the spread of ray actions at the given range.

The formula for the mode amplitude, valid in the nonadiabatic waveguide, has been used to formulate an applicability condition of the adiabatic approximation. In contrast to traditional approaches for solving this problem $[7,8]$ we proceed not from the mode coupling equation but from its solution (albeit approximate). An applicability criterion discussed in the present paper takes into account accumulation of errors with range.

All results are obtained for the wave field that obeys the parabolic equation. This assumption is not necessary: the results are readily generalized to the field governed by the Helmholtz equation. We use the parabolic equation approximation for two reasons. First, unlike the Helmholtz equation it can be easily solved numerically which is very important for testing our approximate formulas. Besides, the parabolic equation formally coincides with the Schrödinger equation and therefore formulas derived in this work may be applied in quantum mechanics to study the motion of a particle in a potential well with time-dependent parameters.

In the present paper we investigate general properties of wave propagation in a range-dependent waveguide by the example of the underwater sound channel. This choice is caused by interests of the authors and by the fact that the topics addressed here are of importance in underwater acoustics [7-10].

The paper is organized as follows. Section II provides a brief description of the ray representation of the wave field. Main relations of the Hamiltonian formalism expressed in terms of both position-momentum and actionangles variables are presented. In Sec. III we consider the mode representation (in the WKB approximation) of the wave field and the mode coupling equation. We also outline a traditional method for estimating the validity region of the adiabatic approximation by using the perturbation theory for the mode coupling equation. In Sec. IV formulas expressing mode amplitudes through parameters of ray trajectories are derived. Section $\mathrm{V}$ focuses on connection between variations of the action variable and mode coupling. It is shown how a standard ray tracing can be used to estimate the number of interacting modes. An applicability criterion for the adiabatic approximation is formulated as restriction on the spread of ray actions. These results are verified and illustrated using numerical examples. The conclusions are summarized in the final section.

\section{RAY REPRESENTATION OF THE WAVE FIELD}

\section{A. Parabolic equation approximation}

Consider a monochromatic wave field at a carrier frequency $f$ in a two-dimensional acoustic waveguide with the sound speed $c$ being a function of depth, $z$, and range, $r$. We shall assume that at each range point the sound speed profile has a single minimum. The wave field $u(r, z)$ is governed by the Helmholtz equation $[7,8,11]$

$$
\frac{1}{r} \frac{\partial}{\partial r} r \frac{\partial u}{\partial r}+\frac{\partial^{2} u}{\partial z^{2}}+\frac{\Omega^{2}}{c^{2}(r, z)} u=0,
$$

where $\Omega=2 \pi f$. Select a reference sound speed $c_{0}$ such that $\left|c(r, z)-c_{0}\right|<<c_{0}$ (in underwater acoustics such 
choice of $c_{0}$ is always possible) and assume that grazing angles of the propagating waves are small. Then it is convenient to introduce an envelope function $v(r, z)$ connected to $u(r, z)$ by an expression

$$
u=\frac{v}{\sqrt{r}} e^{i k r}
$$

with $k=\Omega / c_{0}$. This function is approximately described by the standard parabolic equation $[7,11,12]$

$$
2 i k \frac{\partial v}{\partial r}+\frac{\partial^{2} v}{\partial z^{2}}-2 k^{2} U v=0
$$

where

$$
U(r, z)=\frac{1}{2}\left(1-\frac{c_{0}^{2}}{c^{2}(r, z)}\right) .
$$

Notice that Eq. (3) formally coincides with the timedependent Schrödinger equation. In this analogy $r, k^{-1}$, and $U(r, z)$ play roles of time, Planck's constant, and potential, respectively.

\section{B. Hamiltonian formalism in terms of momentum-position variables}

In the geometrical optics approximation the wave field is formed by contribution from all rays (eigenrays) arriving at the observation point. The ray trajectory is defined by the Hamilton equations $[6,12]$

$$
\frac{d z}{d r}=\frac{\partial H}{\partial p}, \quad \frac{d p}{d r}=-\frac{\partial H}{\partial z}
$$

with the Hamiltonian

$$
H=p^{2} / 2+U
$$

The momentum $p$ is connected to the ray grazing angle $\chi$ through the relation

$$
p=d z / d r=\tan \chi
$$

A contribution to the wave field from an eigenray is presented in the form

$$
v=A e^{i k S}
$$

where $S$ and $A$ are the ray eikonal and amplitude, respectively. The eikonal $S$ is an analog to Hamilton's principal function in classical mechanics and it is given by an integral

$$
S=\int(p d z-H d r)
$$

running over the ray path $[5,12,13]$. An explicit expression for the ray amplitude depends on the source exciting the wave field. In the case of a point source the wave field is determined by Eq. (3) with an initial condition

$$
v(0, z)=\delta\left(z-z_{0}\right)
$$

Then all rays escape the same point $\left(0, z_{0}\right)$ and each trajectory can be labelled by the ray starting momentum $p_{0}$. The amplitude

$$
A=\sqrt{\frac{k}{2 \pi i\left|\partial z / \partial p_{0}\right|}} e^{-i \mu \pi / 2},
$$

where $\mu$ is the Maslov index or integral number of times that the ray passes through caustics [14].

\section{Action-angle variables}

Since ray trajectories in the waveguide are oscillating curves, their analysis may be simplified by using the socalled action-angle variables $[5,6]$. In mechanics the latter are often applied to study oscillations of particles in potential wells.

\section{Range-independent waveguide}

First, consider a range-independent waveguide where the Hamiltonian $H$ remains constant along the ray path (analog to the energy conservation law in mechanics). The action variable $I$ is defined by the integral $[5,6]$

$$
I=\frac{1}{2 \pi} \oint p d z=\frac{1}{\pi} \int_{z_{\min }}^{z_{\max }} d z \sqrt{2[H-U(z)]},
$$

where $z_{\min }$ and $z_{\max }$ are the lower and upper ray turning depths, respectively, satisfying the condition $U(z)=H$. Equation (12) defines the "energy" $H$ as a function of the action $I$. The ray trajectory is a periodic curve whose period along the $r$-axis (the cycle length), $D$, and the angular frequency of spatial oscillations, $\omega$, are determined by the relation

$$
\frac{d H}{d I}=\omega=\frac{2 \pi}{D} .
$$

Take one cycle of the unperturbed ray path that begins at the minimum of the trajectory. At the first half-cycle the canonical transformation,

$$
p=p(I, \theta), \quad z=z(I, \theta),
$$

from the position-momentum, $(p, z)$, to the action-angle, $(I, \theta)$, variables is determined by the equations $[5,6,15]$

$$
p=\frac{\partial G}{\partial z}, \quad \theta=\frac{\partial G}{\partial I},
$$

with

$$
G(I, z)=\int_{z_{\min }}^{z} d z \sqrt{2[H(I)-U(z)]}
$$


being a generating function of the canonical transformation. At this half-cycle the angle variable $\theta$ varies from 0 to $\pi$. The transformation is continued to the next halfcycle $(\pi<\theta<2 \pi)$ using relations $p(I, \theta)=-p(I, 2 \pi-\theta)$, and $z(I, \theta)=z(I, 2 \pi-\theta)$. The ray equations in the new variables take the trivial form

$$
\frac{d I}{d r}=-\frac{\partial H}{\partial \theta}=0, \quad \frac{d \theta}{d r}=\frac{\partial H}{\partial I}=\omega(I) .
$$

Note, that the so defined angle variable $\theta$ varies from 0 to $2 \pi$ at a part of the trajectory beginning at one minimum and ending at the next one. To make the angle variable continuous, its value should be increased by $2 \pi$ at the beginning of each new cycle. Both functions in Eq. (14) are periodic in $\theta$ with period $2 \pi$. Correspondingly, an arbitrary function $F(z)$ expressed through the actionangle variables becomes a periodic function of $\theta$ and can be expanded in a Fourier series

$$
F(z)=\sum_{\nu=0}^{\infty} F_{\nu}(I) \cos \nu \theta
$$

The coefficients $F_{\nu}(I)$ are expressed analytically only for a few special examples of $U(z)$ and $F(z)$. But numerical evaluation of these coefficients can be easily performed using a standard ray code. If we denote the ray trajectory with the action variable $I$ by $z(r)$, then

$$
\begin{gathered}
F_{\nu}(I)=\frac{q_{\nu}}{D(I)} \\
\times \int_{0}^{D(I)} d r F\left(z\left(r_{\min }+r\right)\right) \cos \left(\nu \omega(I)\left(r_{\min }+r\right)\right),
\end{gathered}
$$

where $q_{0}=1, q_{\nu}=2$ for $\nu>0$, and $r_{\min }$ is a range corresponding to a minimum of the ray path.

\section{Range-dependent waveguide}

In a range-dependent waveguide canonical transformations from $(p, z)$ to $(I, \theta)$ variables are different at different range points. At each particular range $r$ the transformation is defined using the so-called reference waveguide: an imaginary range-independent waveguide whose cross-section coincides with that of a real one at the given range. The connection between pairs $(p, z)$ and $(I, \theta)$ at range $r$ is determined by formulas present in Sec. II C 2 that should be applied in a corresponding reference waveguide. Since the functions $p(I, \theta), z(I, \theta)$, $G(I, z), H(I), \omega(I)$, and $F_{\nu}(I)$ become different at different ranges they acquire an additional argument $r$ that labels the reference waveguide.

The canonical transformation is determined by the equation [5]

$$
d S=p d z-H d r=d G-\theta d I-\bar{H} d r
$$

where $\bar{H}$ is a new Hamiltonian. According to this relation

$$
\bar{H}(I, \theta, r)=H(I, r)+\Lambda(I, \theta, r)
$$

with

$$
\Lambda(I, \theta, r)=\left.\frac{\partial G(I, z, r)}{\partial r}\right|_{z=z(I, \theta, r)}
$$

The Hamilton equations retains their canonical form

$$
\frac{d I}{d r}=-\frac{\partial \bar{H}}{\partial \theta}=-\frac{\partial \Lambda}{\partial \theta}, \frac{d \theta}{d r}=\frac{\partial \bar{H}}{\partial I}=\omega+\frac{\partial \Lambda}{\partial I},
$$

where

$$
\omega(I, r)=\frac{\partial H(I, r)}{\partial I} .
$$

The ray eikonal in the new variables can be found by integrating Eq. (20). After some algebra we get

$$
\begin{gathered}
S=\int_{0}^{r}(I d \theta-(H+\Lambda) d r) \\
-G(z(0), I(0), 0)+G(z(r), I(r), r) \\
+\theta(0) I(0)-\theta(r) I(r) .
\end{gathered}
$$

Initial and final values of the angle variable, $\theta(0)$ and $\theta(r)$, present in the right hand side should be taken modulo $2 \pi$.

The term $\Lambda$ can be expressed through coefficients of an expansion

$$
\left.\frac{\partial U(r, z)}{\partial r}\right|_{z=z(I, \theta, r)}=\sum_{\nu=0}^{\infty} V_{\nu}(I, r) \cos \theta
$$

analogous to Eq. (18). First, notice that

$$
\begin{aligned}
\frac{\partial}{\partial r} G(I, z, r) & = \pm \int_{z_{\min }}^{z}\left(\frac{\partial H(I, r)}{\partial r}-\frac{\partial U(r, z)}{\partial r}\right) \\
& \times \frac{d z}{\sqrt{2[H(I, r)-U(r, z)]}}
\end{aligned}
$$

where \pm denotes the sign of $p$ at a current half-cycle. From Eq. (12) it follows that

$$
\begin{gathered}
\frac{\partial H(I, r)}{\partial r}=\frac{\omega(I, r)}{\pi} \\
\times \int_{z_{\min }}^{z_{\max }} \frac{\partial U(r, z)}{\partial r} \frac{d z}{\sqrt{2[H(I, r)-U(r, z)]}} .
\end{gathered}
$$


The right hand side, formally, can be considered as an integral over a half-cycle of the ray path in the reference waveguide. Then

$$
\frac{\omega(I, r) d z}{\sqrt{2[H(I, r)-U(r, z)]}}=d \theta
$$

and

$$
\frac{\partial H(I, r)}{\partial r}=V_{0}(I, r) .
$$

Similarly it can be shown that

$$
\begin{aligned}
& \int_{z_{\min }}^{z} \frac{\partial U(r, z)}{\partial r} \frac{d z}{\sqrt{2[H(I, r)-U(r, z)]}} \\
= & \frac{1}{\omega}\left(\theta V_{0}(I, r)+\sum_{\nu=1}^{\infty} V_{\nu}(I, r) \frac{\sin (\nu \theta)}{\nu}\right) .
\end{aligned}
$$

Combining Eqs. (27), (29), and (30) we arrive at

$$
\Lambda=-\frac{1}{\omega} \sum_{\nu=1}^{\infty} V_{\nu}(I, r) \frac{\sin (\nu \theta)}{\nu} .
$$

Note that the coefficients $V_{\nu}(I, r)$ can be calculated using Eq. (19) in a reference waveguide corresponding to range $r$ with $F$ replaced by $V(r, z)=\partial U(r, z) / \partial r$. When evaluating the right hand side of Eq. (19) one should integrate over the ray path computed in the reference waveguide but not in the real one. In this calculation the argument $r$ of function $V(r, z)$ must be considered as a constant labelling the reference waveguide.

The adiabatic approximation for rays is valid if the sound speed $c$ is a so slow function of range that $\Lambda$ becomes negligible. Then the action variable $I$ remains constant along the ray path $[5,7]$.

\section{MODE REPRESENTATION IN THE WKB APPROXIMATION}

\section{A. Eigenfunctions and eigenvalues}

The normal mode representation of the wave field in a range-independent waveguide is given by an expansion into a sum of eigenfunctions of the Sturm-Liouville eigenvalue problem $[8,16]$

$$
\frac{1}{2} \frac{d^{2} \varphi_{m}}{d z^{2}}+k^{2}\left(H_{m}-U\right) \varphi_{m}=0
$$

with appropriate boundary conditions. The latter can be determined by reflection coefficients at the surface, $V_{s}=e^{i \phi_{s}}$, and at the bottom, $V_{b}=e^{i \phi_{b}}$. We assume that reflections at boundaries occur without energy loss and therefore $\phi_{s}$ and $\phi_{b}$ are real constants. In particular, for a pressure release surface and rigid bottom we have $\phi_{s}=\pi$ and $\phi_{b}=0$. In case when the upper (lower) mode turning points lie within a water bulk we have $\phi_{s}=-\pi / 2$ $\left(\phi_{b}=-\pi / 2\right)$. The eigenfunctions are orthogonal and normalized in such a way that

$$
\int d z \varphi_{m} \varphi_{n}=\delta_{m n}
$$

In the WKB approximation the eigenvalue of the $m$-th mode is

$$
H_{m}=H\left(I_{m}\right)
$$

where $I_{m}$ satisfies the quantization rule $[7,16]$

$$
k I_{m}=m-\frac{\phi_{s}+\phi_{b}}{2 \pi} .
$$

The quantity $I_{m}$ can be treated as an action variable associated with the $m$-th mode: if only this mode is excited then all rays have the same action $I=I_{m}$. Using Eq. (13) we get a simple approximate relation for the difference between eigenvalues of neighboring modes [7]

$$
\frac{d H_{m}}{d m}=H_{m+1}-H_{m}=\omega_{m} / k,
$$

where $\omega_{m} \equiv \omega\left(I_{m}\right)$.

The $m$-th eigenfunction $\varphi_{m}(z)$ between its turning points can be represented as [16]

$$
\varphi_{m}(z)=\varphi_{m}^{+}(z)+\varphi_{m}^{-}(z)
$$

where

$$
\begin{gathered}
\varphi_{m}^{ \pm}(z)=Q_{m} e^{\left. \pm i\left(k S_{m}(z)+\phi_{b} / 2\right)\right)} \\
S_{m}(z)=\int_{z_{\min }}^{z} d z p_{m}(z), \quad p_{m}(z)=\sqrt{2\left[H_{m}-U(z)\right]}
\end{gathered}
$$

and

$$
Q_{m}=\sqrt{\frac{\omega_{m}}{2 \pi p_{m}(z)}}
$$

\section{B. Mode coupling equation}

In each cross-section of a waveguide the field can be decomposed into a sum of the local modes, that is the modes of the reference waveguide (see Sec. II C 2) corresponding to this particular range. Since at different range points we deal with, generally, different reference waveguides, the eigenfunctions and eigenvalues become functions of range. The mode representation of the wave field in a range-dependent waveguide has the form

$$
u(r, z)=\sum_{m} a_{m}(r) \varphi_{m}(r, z),
$$


where we have emphasized the $r$ dependence of the eigenfunction. By substituting this expression in Eq. (3) and using the orthogonality condition (33) we get the mode coupling equation

$$
\frac{d a_{m}}{d r}+i k H_{m} a_{m}=-\sum_{m_{1}} a_{m_{1}} \int d z \frac{\partial \varphi_{m_{1}}}{\partial r} \varphi_{m} .
$$

For a point source defined by Eq. (10) it should be solved with an initial condition

$$
a_{m}(0)=\varphi_{m}\left(0, z_{0}\right)
$$

For later convenience we present the derivative $\partial \varphi_{m} / \partial r$ as an expansion

$$
\frac{\partial \varphi_{m}}{\partial r}=\sum_{\nu \neq 0} B_{m \nu} \varphi_{m+\nu}
$$

Substituting this in Eq. (42) yields

$$
\frac{d a_{m}}{d r}+i k H_{m} a_{m}=\sum_{\nu \neq 0} B_{m \nu} a_{m+\nu}
$$

It can be shown that

$$
B_{m \nu}=\frac{1}{H_{m}-H_{m+\nu}} \int d z \varphi_{m} \frac{\partial U}{\partial r} \varphi_{m+\nu} .
$$

This formula is derived by differentiating Eq. (32) for the reference waveguide with respect to $r$ and exploiting the normalization condition (33). A detailed derivation of a more general result for the wave field governed by the Helmholtz equation see in Ref. [8].

\section{Matrix elements}

In the WKB approximation the matrix element of a smooth function $F(z)$ [16]

$$
F_{m \nu}=\int d z \varphi_{m}(z) F(z) \varphi_{\nu}(z)
$$

with $m, n>>1$ can be expressed through coefficients of expansion (18). Indeed, take $F(z)$ whose characteristic scale is much greater than the wavelength $2 \pi / k$. Then the matrix elements are very small unless $|m-\nu|<<m$. Making use of Eqs. (37)-(39) rewrite Eq. (47) in the form

$$
\begin{gathered}
F_{m, m+\Delta m}=2 \int_{z_{\min }}^{z_{\max }} d z F(z) Q_{m}^{2}(z) \\
\quad \times \cos \left[k\left(S_{m+\Delta m}(z)-S_{m}(z)\right)\right] .
\end{gathered}
$$

From Eqs. (36) and (39) it follows that

$$
k\left(S_{m+\Delta m}(z)-S_{m}(z)\right)=\omega_{m} \Delta m \int_{z_{\min }}^{z} \frac{d z}{p_{m}(z)} .
$$

According to Eq. (7) $d z / p_{m}=d r$ and the integral on the right can be considered as a shift along the $r$-axis between a minimum and a current point of a ray trajectory with action $I_{m}$. In the range-independent waveguide $\omega_{m} r=$ $\theta$ and we get a desired connection between the matrix elements and the coefficients of Eq. (18)

$$
\begin{gathered}
F_{m, m+\Delta m}=\frac{1}{2 \pi} \int_{0}^{2 \pi} d \theta F\left(z\left(I_{m}, \theta\right)\right) \cos (\Delta m \theta) \\
=\frac{1}{2} F_{|\Delta m|}\left(I_{m}\right) .
\end{gathered}
$$

According to this result Eq. (46) in the high frequency approximation reduces to

$$
B_{m \nu}=-\frac{k}{2 \nu \omega_{m}} V_{|\nu|}\left(I_{m}, r\right)
$$

\section{Adiabatic approximation for mode amplitudes}

In the adiabatic approximation the right hand side of the mode coupling equation (45) is assumed to be negligible $[7,8]$ and we get

$$
a_{m}(r)=a_{m}(0) e^{-i k \int_{0}^{r} H_{m}\left(r^{\prime}\right) d r^{\prime}}
$$

The first order correction can be obtained using a simple perturbation theory. Consider the case when only one mode with number $m_{0}$ is excited at $r=0$, that is

$$
a_{m}(0)=\delta_{m m_{0}}
$$

Substituting Eq. (52) with $a_{m}(0)$ defined by Eq. (53) into the right hand side of Eq. (45) we find that for $m \neq m_{0}$

$$
a_{m}(r)=e^{-i k \int_{0}^{r} H_{m_{0}}\left(r^{\prime}\right) d r^{\prime}} q_{m}
$$

where

$$
q_{m}=\int_{0}^{r} d r^{\prime} B_{m, m_{0}-m}\left(r^{\prime}\right) e^{i\left(m-m_{0}\right) \int_{0}^{r^{\prime}} \omega_{m_{0}}\left(r^{\prime \prime}\right) d r^{\prime \prime}} .
$$

At high frequencies, when Eq. ( 51) is valid, $q_{m}$ can be presented in the form

$$
\begin{gathered}
q_{m}=-\frac{k}{2\left(m-m_{0}\right)} \\
\times \int_{0}^{r} \frac{d r^{\prime}}{\omega_{m}\left(r^{\prime}\right)} V_{\left|m-m_{0}\right|}\left(r^{\prime}, I_{m}\right) e^{i\left(m-m_{0}\right) \int_{0}^{r^{\prime}} \omega_{m_{0}}\left(r^{\prime \prime}\right) d r^{\prime \prime}} .
\end{gathered}
$$

The condition

$$
\left|q_{m}\right|<<1
$$


(or its analog for the Helmholtz equation) is traditionally considered as a starting point for studying the applicability of adiabatic approximation $[7,8]$. In particular it turns out that the inequality (57) requires that

$$
D / L<<1,
$$

where $L$ is the characteristic scale of the horizontal range dependence. Another criterion involving the dependence on a frequency of the propagating wave has the form $[8,17]$

$$
k D^{2} / L<<1 .
$$

There are less general (but less restrictive and therefore more useful) criteria that can be obtained for waveguides with some special properties [8].

\section{PROJECTION OF THE RAY REPRESENTATION ONTO NORMAL MODES}

\section{A. Mode amplitude as a function of ray parameters}

In this section we show that the mode amplitude in a range-dependent waveguide can be approximately expressed through parameters of ray trajectories. In more details this issue has been considered in Refs. [1, 2]. Our starting point is an equation

$$
a_{m}(r)=\int d z v(r, z) \varphi_{m}(r, z)
$$

that follows from the mode orthogonality condition (33). Substituting $v(r, z)$ from Eq. (8) and using the WKB approximation for $\varphi_{m}(r, z)$ we get $a_{m}=a_{m}^{+}+a_{m}^{-}$with

$$
a_{m}^{\sigma}=\int d z B(r, z, \sigma) e^{i k \Phi(r, z, \sigma)},
$$

where $\sigma$ denotes plus or minus,

$$
B=e^{-i \mu \pi / 2+i \sigma \phi_{b} / 2} A Q_{m},
$$

and

$$
\Phi=S+\sigma S_{m} .
$$

In the high frequency approximation (large $k$ ) integral (61) can be evaluated using a standard stationary phase technique [13]. This yields

$$
\begin{gathered}
a_{m}^{\sigma}=\left.\sqrt{\frac{2 \pi}{k\left|\partial^{2} \Phi / \partial z^{2}\right|}}\right|_{z=z_{s t}} \\
\left.B \exp \left[i k \Phi+i \frac{\pi}{4} \operatorname{sgn}\left(\partial^{2} \Phi / \partial z^{2}\right)\right]\right|_{z=z_{s t}},
\end{gathered}
$$

with $z_{s t}$ being a stationary phase point where the derivative $\partial \Phi / \partial z$ vanishes. The function $\operatorname{sgn}(x)$ gives the sign of its argument. In classical mechanics it is well-known that the eikonal $S$ considered as a function of range $r$, starting coordinate $z_{0}$, and a final coordinate $z$ obeys the relation [5]

$$
\frac{\partial S}{\partial z}=p
$$

where $p$ is a momentum at range $r$. Combining this with Eq. (39) we get

$$
\frac{\partial \Phi}{\partial z}=p+\sigma p_{m}
$$

This derivative vanishes at a point $z$ such that a ray arriving at this point has an action

$$
I=I_{m}
$$

and

$$
\sigma=-\operatorname{sgn}(p) .
$$

The condition (67) singles out rays whose actions at the given range $r$ are equal to the action of the $m$-th mode. In quantum theory a similar result was obtained in Ref. [4].

For the second derivative of $\Phi$ we have an expression

$$
\begin{gathered}
\frac{\partial^{2} \Phi}{\partial z^{2}}=\frac{\partial p}{\partial z}-\sigma \frac{\partial U / \partial z}{p_{m}} \\
=\frac{1}{p} \frac{\partial H}{\partial z}=\frac{\omega}{p} \frac{\partial I}{\partial z},
\end{gathered}
$$

where $H$ and $I$ are considered as functions of the ray arrival depth at the given range $r$. From Eq. (64) it follows that

$$
\begin{gathered}
a_{m}^{\sigma}=\left.A|k \partial I / \partial z|^{-1 / 2}\right|_{z=z_{s t}} \\
\left.\exp (i k \Phi-i \pi / 4 \sigma \operatorname{sgn}(\partial I / \partial z))\right|_{z=z_{s t}} .
\end{gathered}
$$

To complete the calculation of the mode amplitude we should express the derivative $\partial I / \partial z$ through parameters of rays. In the next subsection this is done for two important examples of the starting field.

\section{B. Point source and source exciting a single mode}

In the case of a point source forming the starting field (10) we deal with a congruence of rays leaving point $\left(0, z_{0}\right)$. Labelling each ray by its starting momentum $p_{0}$ we present the derivative $\partial I / \partial z$ as $\left(\partial I / \partial p_{0}\right) /\left(\partial z / \partial p_{0}\right)$. Then insertion of Eq. (11) into Eq. (70) yields

$$
a_{m}^{\sigma}=\frac{1}{\sqrt{2 \pi\left|\partial I / \partial p_{0}\right|}}
$$




$$
\times \exp \left(k\left(S+\sigma S_{m}\right)-i \mu \pi / 2+i \sigma \phi_{b} / 2+i(\beta-1) \pi / 4\right),
$$

with

$$
\beta \equiv-\sigma \operatorname{sgn}\left(\partial I / \partial p_{0}\right) \operatorname{sgn}\left(\partial z / \partial p_{0}\right)
$$

Formula (71) accounts for a contribution from a ray satisfying the condition (67) to the $m$-th mode. The mode amplitude is evaluated by summing up contributions from all such rays. Note that in the range-independent waveguide at any range point and for any mode there are exactly two rays satisfying Eq. (67) [1-3, 18]. They escape the source at grazing angles (equal in absolute value and opposite in sign) that coincides with grazing angles of the quasi-plane waves $\varphi_{m}^{+} e^{-i k H_{m} r}$ and $\varphi_{m}^{-} e^{-i k H_{m} r}$ at depth $z_{0}$. This statement remains valid in an adiabatic waveguide as well. Notice that if rays are adiabatic, i.e. the term $\Lambda$ in the Hamiltonian (21) is negligible, then Eq. (71) reduces to Eq. (52) with $a_{m}(0)$ defined by Eq. (43). A corresponding transformation is rather simple but somewhat lengthy and therefore we do not present it here.

A similar result can be derived for a source exciting a single mode with $m=m_{0}$ [1]. In this case we have two congruences of rays associated with functions $\varphi_{m_{0}}^{ \pm}$. Initial actions of all rays are equal to $I_{m_{0}}$ and their starting depths are located between turning points of the mode. There are two rays escaping each point $z_{0}$ within this interval of depths. These rays have starting momenta

$$
p_{0}= \pm \sqrt{2\left(H_{m_{0}}(0)-U\left(z_{0}\right)\right)}
$$

and complex amplitudes [1, 19]

$$
A=\frac{Q_{m_{0}}\left(z_{0}\right)}{\sqrt{\left|\partial z / \partial z_{0}\right|}} e^{ \pm i\left(S_{m_{0}}(z)+\phi_{b} / 2\right)} .
$$

Here we label rays by their starting depths. Substituting Eq. (74) into Eq. (70) and representing $\partial I / \partial z$ as $\left(\partial I / \partial z_{0}\right) /\left(\partial z / \partial z_{0}\right)$ yields

$$
\begin{gathered}
a_{m}^{\sigma}=\frac{Q_{m_{0}}\left(z_{0}\right)}{\sqrt{k\left|\partial I / \partial z_{0}\right|}} \\
\times \exp \left(i k\left(S+\sigma S_{m}+\beta_{0} S_{m_{0}}\left(z_{0}\right)\right)\right) \\
\times \exp \left(-i \mu \pi / 2+i \sigma \phi_{b} / 2+i \beta \pi / 4\right),
\end{gathered}
$$

with

$$
\beta \equiv-\sigma \operatorname{sgn}\left(\partial I / \partial z_{0}\right) \operatorname{sgn}\left(\partial z / \partial z_{0}\right), \quad \beta_{0}=\operatorname{sgn}\left(p_{0}\right)
$$

An important cautionary remark should be made. When using the stationary phase technique we assume that the integration in Eq. (61) goes over an interval exceeding

$$
\delta z=\sqrt{\frac{2 \pi}{k\left|\frac{\partial^{2} \Phi}{\partial z^{2}}\right|}} .
$$

Therefore our approach requires that

$$
\delta z<<\Delta z
$$

where $\Delta z$ is a difference between mode turning points. Denote by $\Delta I$ a spread of the ray action at the range of observation. A corresponding spread of the "energy" $H$ in accord with Eqs. (13) and (24) is $\Delta H=2 \pi \Delta I / D$. Assuming that the spread of ray depths at the range of observation has the same order of magnitude as $\Delta z$ and using Eq. (69) we obtain an order-of-magnitude estimate

$$
\frac{\partial^{2} \Phi}{\partial z^{2}} \approx \frac{1}{p} \frac{\Delta H}{\Delta z}=\frac{1}{p} \frac{2 \pi}{D} \frac{\Delta I}{\Delta z},
$$

where $p$ is an rms value of momentum at range $r$. As it follows from Eq. (67) an initially excited mode breaks up into a group of approximately

$$
\Delta m=k \Delta I
$$

modes. Using a rough estimate $p D / 2 \approx \Delta z$ and combining Eqs. (77), (79), and (80) we get

$$
\delta z \approx \frac{\Delta z}{\sqrt{\Delta m / 2}} .
$$

The condition (78) is met only if $\Delta m>>1$, that is if many modes are excited. Thus, Eq. (75) is valid only in a strongly nonadiabatic environment and at long enough ranges where an initially excited mode breaks up into many modes. In contrast, a point source usually excites a large number of modes and therefore the condition (78) practically does not restrict applicability of Eq. (71).

\section{VARIATIONS OF ACTION VARIABLE AND MODE COUPLING}

\section{A. Number of interacting modes and applicability of adiabatic approximation}

First, consider the case when only one mode is excited at $r=0$. In spite of the remark made at the end of Sec. IV B the condition (67) even at short ranges properly indicates rays contributing to the given mode. Therefore Eq. (80) provides an estimate for the number of interacting modes valid at any range. Correspondingly, the applicability condition of adiabatic approximation can be formulated in the form

$$
\Delta I<<1 / k
$$

The quantity $\Delta I$ on the left represents a difference between initial and final actions along a typical ray. Substituting Eq. (31) into the first of ray equations (23) yields

$$
\Delta I=I(r)-I_{m_{0}}
$$




$$
=-\frac{1}{\omega} \sum_{\nu=1}^{\infty} \int_{0}^{r} d r V_{\nu}\left(I_{m_{0}}, r\right) \cos (\nu \theta) .
$$

In the integrands on the right we neglect the deviation of action from its starting value. In the same approximation the second of ray equations (23) gives

$$
\theta=\theta_{0}+\int_{0}^{r} \omega\left(I_{m_{0}}, r\right) d r
$$

Substituting this into Eq. (83) we see that Eq. agrees with the condition (57).

Since we consider a linear problem and the wave field can be always represented as a superposition of normal modes the criterion (82) remains valid for an arbitrary source. But in the case of a point source we have an explicit expression for the mode amplitude (Eq. (71)) which can be used at both short ranges where the adiabatic approximation is still valid and at long ranges where this approximation fails. Proceeding from this result we shall derive a more accurate criterion. It is natural to expect that the deviation from the adiabaticity first reveals itself in a deviation of the phase $k \Phi$ defined by Eqs. (63), (67), and (68) from its value obtained under assumption that $\Lambda$ can be dropped.

In order to estimate this phase deviation take a ray (we shall call it nonadiabatic) contributing to the $m$-th mode and compare its phase with that of a similar ray (adiabatic) whose trajectory satisfies Eqs. (23) with $\Lambda=0$. Both rays escape the same point source and at range $r$ have the same value of action $I=I_{m}$. Formally, we shall assume that $\Lambda$ is proportional to some small parameter $\varepsilon$. Our purpose is to evaluate the difference between phases of the rays up to terms of order $O(\varepsilon)$. We shall use the symbols $\delta S, \delta I, \delta \theta$, and $\delta z$ to denote the difference in eikonals, action variables, angle variables, and vertical coordinates of the nonadiabatic and adiabatic rays, respectively.

Compare eikonals (see Eq. (25)) of our rays. From Eqs. (15) it follows that

$$
G\left(z_{0}, I_{m}+\delta I_{0}, 0\right)-G\left(z_{0}, I_{m}, 0\right)=\theta(0) \delta I(0),
$$

and

$$
G\left(z+\delta z(r), I_{m}, r\right)-G\left(z, I_{m}, r\right)=p_{m} \delta z(r) .
$$

Making use of ray equations (23) yields

$$
\begin{gathered}
\delta \int_{r_{0}}^{r_{1}}(I d \theta-H d r) \\
=\int_{r_{0}}^{r_{1}}\left(I_{m} d \delta \theta+\delta I d \theta-\frac{\partial H}{\partial I} \delta I d r\right) \\
=I_{m}(\delta \theta(r)-\delta \theta(0)) .
\end{gathered}
$$

Combining Eqs. (86) and (87) we find

$$
\delta S=p_{m} \delta z(r)-\int_{r_{0}}^{r_{1}} \Lambda d r .
$$

The integration goes along the adiabatic ray. We assume that the nonadiabaticity is so week that the constants $\mu, \beta$, and $\sigma$ for both rays are the same. According to Eq. (39) the difference between $S_{m}$ corresponding to our rays can be estimated as $\delta S_{m}=p_{m} \delta z(r)$. Taking into account Eq. (68) we finally arrive at

$$
\delta \Phi=-k \int \Lambda d r
$$

A slightly different derivation of Eq. (89) is given in Ref. [3]. Thus, applicability of adiabatic approximation requires that

$$
\left|k \int \Lambda d r\right| \ll \pi
$$

Using an explicit expression for $\Lambda$ given by Eq. (31) and approximating $\theta$ by expression presented in Eq. (84) it is not difficult to show that the condition (90) agrees with Eqs. (56) and (57).

The criterion (82) is much more convenient for practical applications than (57) and (90). Indeed, evaluation of $\Delta I$ can be performed using a standard ray code without exploiting formula (83). To find action $I$ at the given range $r$ one should (i) compute the ray parameters $p$ and $z$ at this range and (ii) using them as initial conditions evaluate (with the same ray code) integral (12) over the ray cycle in a corresponding reference waveguide.

\section{B. Examples}

To verify and illustrate the above results we have computed wave fields in two range-dependent hydroacoustic waveguides. This is done using the code MMPE [20] originally created for solving the wide angle parabolic equation. It has been slightly modified to use the standard parabolic equation approximation. All numerical results presented in this section have been obtained for monochromatic wave fields at a carrier frequency of 200 Hz.

In our first example the sound speed field is taken in the form $c(r, z)=\bar{c}(z)+\delta c(r, z)$. A range independent constituent

$$
\bar{c}(z)=c_{0}\left(1+\varepsilon\left(e^{2\left(z-z_{a}\right) / B}-2\left(z-z_{a}\right) / B-1\right)\right),
$$

with $c_{0}=1.5 \mathrm{~km} / \mathrm{s}, B=1 \mathrm{~km}, z_{a}=-1 \mathrm{~km}$, and $\varepsilon=0.0057$ represents the so-called Munk profile widely used to study sound propagation in deep sea [7, 11]. We consider a strong range-dependent perturbation modelling a synoptic eddy

$$
\delta c(r, z)=c_{2} \exp \left(-\frac{\left(r-r_{2}\right)^{2}}{\Delta r^{2}}-\frac{\left(z-z_{2}\right)^{2}}{\Delta z(r)^{2}}\right),
$$

where

$$
\Delta z(r)=\Delta z_{c}-\Delta z_{v} \exp \left(-\frac{\left(r-r_{v}\right)^{2}}{\Delta r_{v}}\right) .
$$


The following values of parameters have been selected: $c_{2}=-0.01 \mathrm{~km} / \mathrm{s}, r_{2}=300 \mathrm{~km}, z_{2}=-1 \mathrm{~km}, \Delta r=80$ $\mathrm{km}, \Delta z_{c}=0.5 \mathrm{~km}, \Delta z_{v}=0.25 \mathrm{~km}, r_{v}=320 \mathrm{~km}, \Delta r_{v}=$ $20 \mathrm{~km}$. The isolines of the total sound field are shown in Fig. 1.

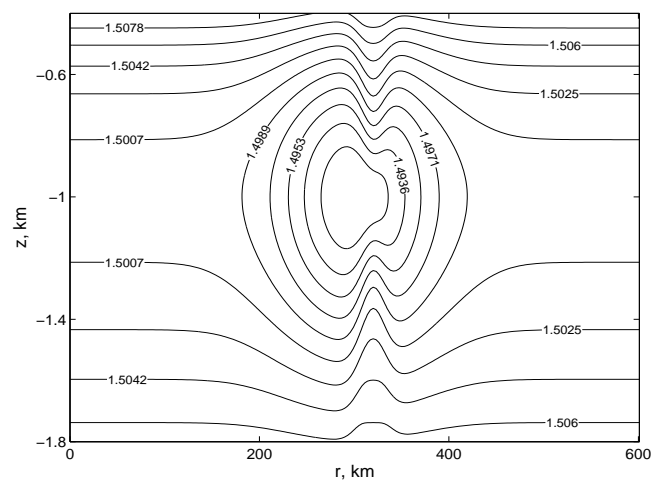

FIG. 1: Sound speed field modelling a synoptic eddy in a deep sea. Contour labels are of sound speed in $\mathrm{km} / \mathrm{s}$.

The upper panel in Fig. 2 shows the deviation of mode amplitude at range $r=600 \mathrm{~km}$ from its starting value at $r=0$. The deviation is taken relative to the rms mode amplitude at $r=0$. It is seen that only modes with $m<15$, whose amplitudes remain practically unchanged, are adiabatic. This fact agrees with predictions following from Eqs. (82) and (90). The middle and lower panels in Fig. 2 present values of $\delta \Phi$ and $k \Delta I=k\left(I(r)-I_{m}\right)$ computed along ray paths escaping the point source with starting values of action variables equal to $I_{m}(m=0, \ldots, 100)$. For each mode there are two such rays with launch angles equal in absolute value and opposite in sign (see comment after Eq. (68)). Solid (dashed) curves in both panels correspond to rays starting upward (downward). Consistent with our expectation, although $\delta \Phi /(2 \pi)$ and $k\left(I-I_{m}\right)$ are not close they have the same order of magnitude and both are very small for adiabatic modes.

The upper panel in Fig. 3 shows the range dependences of mode amplitudes in a situation when a single mode with $m=60$ is excited at $r=0$. Due to scattering at the eddy the 60-th mode breaks up into a group of modes. In order to predict the width of this group using Eq. (80) we have traced a fan of 40 rays with initial actions equal to $I_{60}$. The rays start from 20 points uniformly sampling the depth interval between the turning points of mode 60 . There are two rays with starting momenta defined by Eq. (73) escaping each point. The lower panel of Fig. 3 presents the deviation of action from its starting value as a function of range. It is clearly seen that in agreement with Eq. (80) $k \Delta I$ with $\Delta I=\max \left|I(r)-I_{60}\right|$ representing the spread of actions at the given range, properly predicts the width of the group of excited modes.

In another example of the range-dependent waveguide
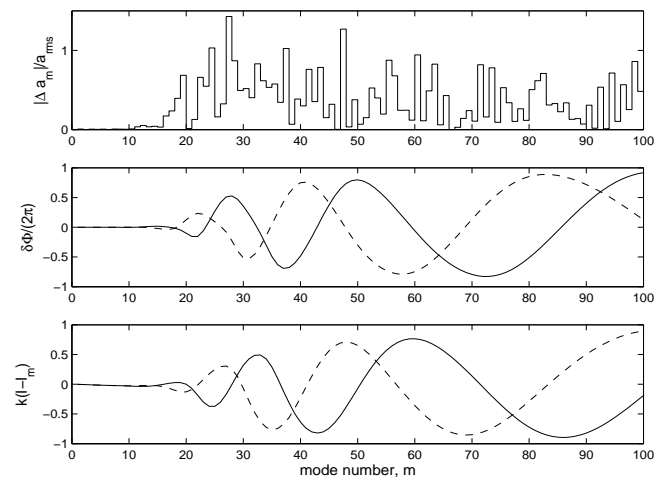

FIG. 2: Modes excited by a point source at a carrier frequency $200 \mathrm{~Hz}$. The source is set at $z=-1 \mathrm{~km}$. Upper panel: Normalized differences between mode amplitudes at ranges $r=0$ and $r=600 \mathrm{~km} ; \Delta a_{m}=a_{m}(600 \mathrm{~km})-a_{m}(0), a_{\mathrm{rms}}$ is a rms mode amplitude at $r=0$. Middle panel: phase variations $\delta \Phi$ due to nonadiabaticity predicted by Eq. (89) at $r=600 \mathrm{~km}$ for rays starting upward (solid line) and downward (dashed line). Lower panel: deviations of ray actions from their starting values $I_{m}$ at $r=600 \mathrm{~km}$ computed for rays starting upward (solid line) and downward (dashed line).
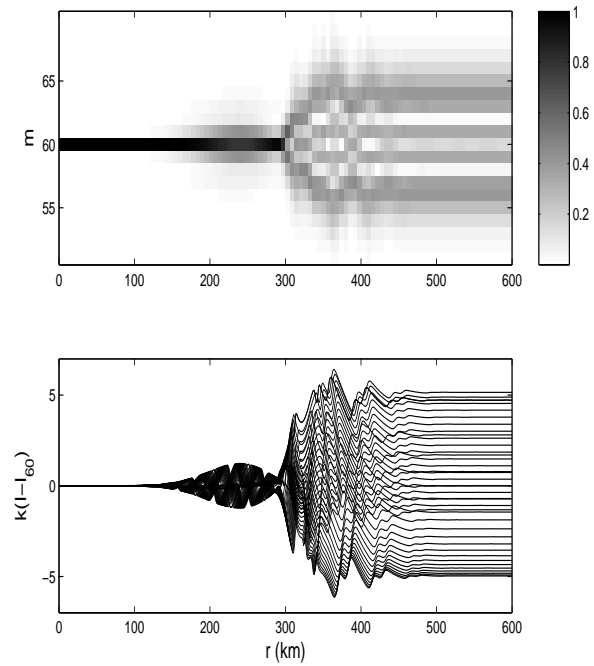

FIG. 3: Upper panel: range dependences of mode amplitudes (shown by gradations of gray) for the case when only one mode with $m=60$ is excited at $r=0$. Lower panel: deviation of the action variable from its starting value computed for a fan of 40 rays starting from different depth points with actions $I(0)=I_{60}$.

the sound speed field

$$
c(r, z)=\frac{R-r}{R} c_{1}(z)+\frac{r}{R} c_{2}(z),
$$

with $R=200 \mathrm{~km}$ being the waveguide length, represents a linear superposition of two profiles $c_{1}(z)$ and $c_{2}(z)$. An evolution of the sound speed profile with range is shown in Fig. 4. In this waveguide the mode coupling is rather 
strong and values of $\delta \Phi$ and $k \Delta I$ (not shown) at range $200 \mathrm{~km}$ are on the order of unity for all modes. To check the applicability of estimate (80) we, once again, have considered the case when only the 60 -th mode is excited at $r=0$. Figure 5 again demonstrates that $k \Delta I$ provides a good estimate for the width of a group of normal modes into which the original mode breaks up. A new feature of function $\Delta I(r)=\max \left|I(r)-I_{60}\right|$ absent in Fig. 3 is an appearance of narrow spots in both panels at ranges of about 47, 94, 141, and $188 \mathrm{~km}$. From the viewpoint of the ray-based approach this phenomenon is related to the fact that the sound speed field (94) is a linear function of range. Since $U(r, z) \simeq\left(c(r, z)-c_{0}\right) / c_{0}$ is an almost linear function of $r$, the derivative $\partial U / \partial r$ is practically range-independent and the same is true of the coefficients $V_{\nu}$ present in Eq. (83). At not very long ranges $\theta \approx \theta_{0}+$ $\omega\left(I_{60}, 0\right) r$ and $\left|I-I_{60}\right|$ in this approximation vanishes at ranges equal to integer multiples of the cycle length of the 60 -th mode. The latter is about $47 \mathrm{~km}$.

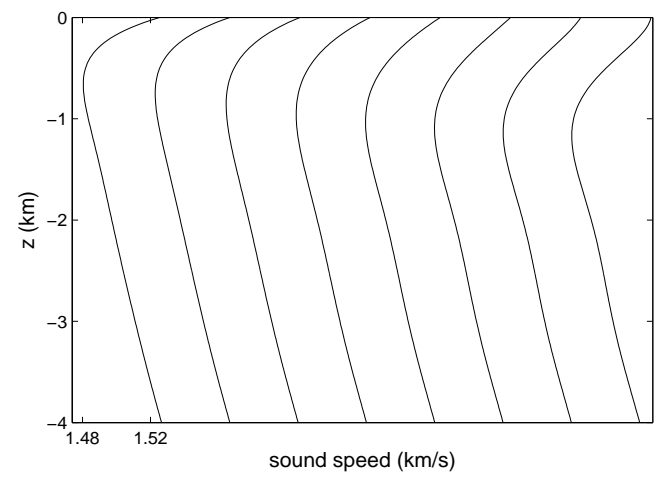

FIG. 4: Sound speed profiles at range points equally spaced within an interval from $r=0$ to $r=200 \mathrm{~km}$ are plotted (from left to right) with a sound speed offset of $0.4 \mathrm{~km} / \mathrm{s}$. The leftmost and rightmost curves represent functions $c_{1}(z)$ and $c_{2}(z)$ in Eq. (94), respectively.
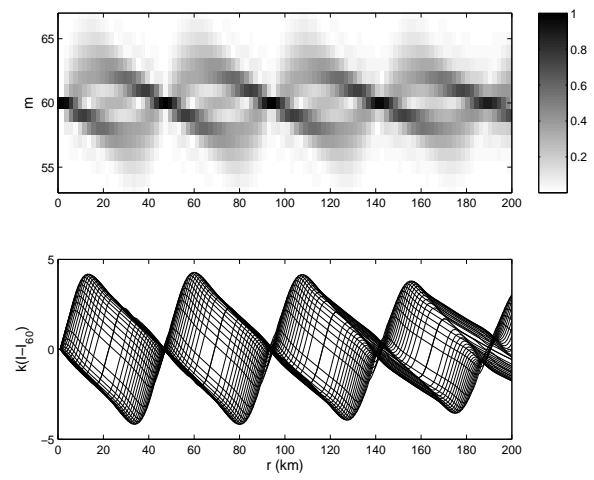

FIG. 5: The same as in Fig. 3 but constructed for a waveguide with the sound speed field defined by Eq. (94) and shown in Fig. 4 .

\section{CONCLUSION}

In this paper we have considered a ray-based method representing an analog of the geometrical optics for modes. In the scope of this approach the mode amplitude is expressed through solutions of the ray equations. It turns out that the $m$-th mode is formed by contributions from rays whose action variables at the range of observation, up to a multiplicative constant, are equal to $m$. Since the ray method remains valid in the nonadiabatic waveguide, the relatively simple formulas connecting rays and modes provide a convenient tool for studying the applicability of adiabatic approximation. In the present paper we have used this option.

Our simplest criterion is given by Eq. (82) that imposes limitations on the range variation of the action variable. This equation establishes connection between the validity of adiabatic approximations for rays and modes. It is interesting that when the condition (82) fails, the quantity $k \Delta I$ estimates a number of interacting modes. For the case of a point source we have derived a more accurate criterion (90) whose implementation, however, requires more detailed calculations. Nevertheless, it should be emphasized that (i) the left hand sides of both Eqs. (82) and (90) can be evaluated using a standard ray tracing and (ii) both criteria agree with the condition (57) derived from the mode coupling equation. Note also that unlike conditions (58) and (59), our criteria account for accumulation of errors with range.

Since the left hand sides of Eqs. (82) and (90) are proportional to the carrier frequency it is clear that the lower the frequency, the better these inequalities are satisfied and, hence, the wider the validity region of the adiabatic approximation. The same conclusion follows from Eq. (59).

Finally, notice that all our results have been obtained in the high frequency approximation which, naturally, restricts their generality. In contrast, the use of the parabolic equation approximation is not principal. The results can be easily generalized to the case when the wave field is governed by the Helmholtz equation. Although we have considered sound waves propagating in underwater acoustic waveguides the formulas derived here can be applied for description of wave propagation in different waveguide media and for analysis of a quantum particle oscillating in a potential well with timedependent parameters.

This work was supported by the Russian Foundation for Basic Research under Grant No. 03-02-17246. 
[1] A.L. Virovlyansky and G.M. Zaslavsky, "Wave chaos in terms of normal modes" Phys. Rev. E 59, 1656-1668 (1999).

[2] A.L. Virovlyanskii, L.Ya. Lyubavin, and S.A. Stromkov, "The ray approach for analyzing the modal structure of the sound field in a range-dependent waveguide" Acoustical Physics 47, 517-523 (2001).

[3] A.L. Virovlyansky, A.Yu. Kazarova, and L.Ya. Lyubavin, "Variations of mode amplitudes in a range-dependent waveguide" Acoustical Physics 50, 20-29 (2004).

[4] G.P. Berman and G.M. Zaslavsky, "Condition of stochasicity in quantum nonlinear systems" Physica A 97, 367382 (1979).

[5] L.D. Landau and E.M. Lifshitz, Mechanics, Pergamon Press, Oxford, 1976.

[6] S.S. Abdullaev and G.M. Zaslavsky, "Classical nonlinear dynamics and chaos of rays in wave propagation problems in inhomogeneous media" Sov. Phys. Usp. 38, 645-687 (1991).

[7] L.M. Brekhovskikh and Yu.P. Lysanov, Fundamentals of Ocean Acoustics, Springer-Verlag, Berlin, 1991.

[8] L.M. Brekhovskikh and O.A. Godin, Acoustics of Layered Media. II: Point Sources and Bounded Beams, Springer-Verlag, Berlin, 1999.

[9] E.C. Shang, "Ocean acoustic tomography based on adiabatic mode theory" J. Acoust. Soc. Am. 85, 1531-1537 (1989).

[10] O.A. Godin, "Coupled-mode sound propagation in a range-dependent moving fluid" J. Acoust. Soc. Am. 111, 1984-1995 (2002).
[11] F.B. Jensen, W.A. Kuperman, M.B. Porter, and H. Schmidt, Computational Ocean Acoustics, AIP, Woodbury, New York, 1994.

[12] J. Simmen, S.M. Flatte, and G.-Y. Wan, "Wavefront folding, chaos, and diffraction for sound propagation through ocean internal waves" J. Acoust. Soc. Am. 102, 239-255 (1997).

[13] M. Born and E. Wolf, Principles of optics, Pergamon Press, Oxford, 1968.

[14] M.C. Gutzwiller, "Phase-integral approximation in momentum space and the bound states of an atom" J. Math. Phys. 8, 1979-2000 (1967).

[15] S.S. Abdullaev, Chaos and dynamics of rays in waveguide media, Edited by G. Zaslavsky, Gordon and Breach science publishers, New York, 1993.

[16] L.D. Landau and E.M. Lifshitz, Quantum mechanics, Pergamon Press, Oxford, 1977.

[17] D.M. Milder, "Ray and wave invariants for sofar channel propagation" J. Acoust. Soc. Am. 46, 1259-1263 (1969).

[18] A.L. Virovlyansky, V.V. Kurin, N.V. PronchatovRubtsov, and S.I. Simdyankin, "Fresnel zones for modes" J. Acoust. Soc. Am. 101, 163-173 (1995).

[19] V.P. Maslov and M.V. Fedoriuk, Semi-classical approximation in quantum mechanics, Reidel, Boston, 1981.

[20] K.B. Smith, "Convergence, stability, and sariability of shallow water acoustic predictions using the split-step fourier parabolic equation model" J. Comp. Acoust. 9, 243-285 (2001). 